\title{
Pulsed high dose dexamethasone therapy in chronic immune thrombocytopenic purpura (IIP)
}

\author{
D H Karunatilake ${ }^{1}$, G W D S Herath ${ }^{2}$ \\ Sri Lanka Journal of Child Health, 2001; 30: 69-71
}

(Key words: pulsed high dose dexamethasone therapy, chronic immune thrombocytopenic purpura)

\begin{abstract}
Chronic IIP is a rare disease in childhood with significant management problems for the paediatrician. Several therapeutic modalities are available but most of them are not very applicable to us either due to financial constraints or to risks involved. We have shown that high dose pulsed dexamethasone therapy is effective in the treatment of chronic ITP in children but the numbers involved in our study are too small to draw any firm conclusions.
\end{abstract}

\section{Introduction}

Immune Thrombocytopenic Purpura (ITP) affects approximately 4 in $100.000^{1}$ population per year. The majority undergo complete recovery within six months from diagnosis ${ }^{2}$. Those who continue to have persistent thrombocytopenia for more than six months from the first diagnosis are considered as having chronic ITP. These patients run the risk of having a life threatening bleed although serious intracranial haemorrhage is less common. It is more common to have repeated epistaxis, ecchymoses or petechiae, often associated with viral infections. As a result, these children are forced to lead a restricted life style which is often very agonizing to a young child. On the other hand, paediatricians treating these children also feel obliged to embark on some form of treatment regime for fear of severe haemorrhage even though a majority does not need any invasive or vigorous treatment.

There are different treatment modalities with varying degree of success such as intravenous immunoglobulin (IVIG), long term use of low dose steroids, intravenous / intramuscular anti RhD globulin, immunosuppressive therapy, splenectomy and pulsed high dose dexamethasone therapy ${ }^{1,2,3}$.

Cyclical pulsed therapy with a high dose of dexamethasone has been shown to be beneficial in adults but the efficacy of this treatment in children is yet to be established.

${ }^{1}$ Consultant Paediatrician, ${ }^{2}$ Paediatric Registrar, Colombo South Teaching Hospital.

\section{Method}

We treated four children with chronic ITP who were under our care during the past four and a half years, both in the public and private sector, using pulsed dexamethasone when they showed poor response to continuous low dose steroid therapy with or without the initial administration of intravenous immunoglobulin (IVIG).

Informed consent was obtained from the parents prior to starting treatment. Dexamethasone at a dose of $15 \mathrm{mg} / \mathrm{m}^{2}$ was given orally in two divided doses for five consecutive days, once in every four weeks, for a total period of six months. Platelet counts were done on day 1 , day 5, day 28 and at the end of the full course of therapy in each of the four patients and then monthly for a further period of twelve months. The weight and height of each patient was measured at the beginning of treatment and at three monthly intervals. They were also examined closely for possible side effects to dexamethasone during the course of treatment.

\section{Patient 1}

A two and a half year old boy was first diagnosed as having ITP at the age of one year and a complete course of IVIG was given upon diagnosis when his platelet count dropped to dangerously low levels. He made a temporary recovery following the administration of immunoglobulin but continued to have recurrent bruises, ecchymoses and purpura, often precipitated by trivial viral infections, over the next year. He received two courses of oral prednisolone during this period and was kept on a maintenance dose of prednisolone for a three month period after the second course. As the long term prednisolone failed to maintain sustained remission he was commenced on oral dexamethasone $15 \mathrm{mg} / \mathrm{m}^{2} /$ day for five consecutive days repeating once in every 28 days for six cycles. His platelet count prior to starting therapy was $20 \times 10^{9} / \mathrm{L}$ and before the beginning of the second cycle was $62 \times 10^{9} / \mathrm{L}$. His platelet count remained well over $200 \times 10^{9} / \mathrm{L}$ after completion of six cycles and he is still in remission four years after completing the dexamethasone course. 


\section{Patient 2}

This was a four year old girl who continued to have bleeding manifestations similar to the patient described above for a period of one year and two months. She also failed to maintain sustained remission on prednisolone given over varying periods of time following remission induction on three occasions using $2 \mathrm{mg} / \mathrm{kg}$ /day for two weeks followed by every other day therapy for a further one to two weeks before being tailed off to a maintenance dose of 7.5-10 mg every other day. Her platelet counts prior to starting treatment, before the beginning of the second cycle and after completion of treatment were $32 \times 10^{9} / \mathrm{L}, 78 \times 10^{9} / \mathrm{L}$ and $185 \times 10^{9} / \mathrm{L}$ respectively. Her course was completed three years and two months ago and she is still in remission.

\section{Patient 3}

This was a three year old girl who first presented with gum bleeding, purpura and multiple ecchymotic patches following a febrile illness and her condition generated a great deal of anxiety on admission. She too continued to have repeated bleeding manifestations including repeated gum and nose bleeds. She was also given prednisolone as in the previous instances but oral dexamethasone cyclical therapy was commenced nine months after the onset of the initial illness. Her platelet counts prior to starting treatment, before the beginning of the second cycle and at the end of treatment were $40 \times 10^{9} / \mathrm{L}, 68 \times 10^{9} / \mathrm{L}$ and $238 \times 10^{9} / \mathrm{L}$ respectively. She still remains in remission eleven months after completing her course of treatment.

\section{Patient 4}

This 10 year old boy was referred to us when he continued to have excessive and prolonged bleeding following an uncomplicated tooth extraction. On close questioning the mother revealed that her son used to develop frequent bruises especially over the legs and trunk for the past one and a half years but thought they were sustained during the normal course of play and had no sinister meaning. He too was given prednisolone similar to the other children described above and commenced on oral dexamethasone cyclical therapy when he presented with another severe bleed from a carious tooth while on a maintenance dose of prednisolone. His platelet counts before treatment with dexamethasone, at the beginning of the second cycle and at the end of the complete course of treatment were $22 \times 10^{9} / \mathrm{L}$, $72 \times 10^{9} / \mathrm{L}$ and $202 \times 10^{9} / \mathrm{L}$ respectively. However, he relapsed after being in remission for a period of three years.

A firm diagnosis of ITP was made in each case using standard investigative procedure including bone marrow examination prior to the administration of steroids. Only patient No.1 who was managed in the private sector had the benefit of IV immunoglobulin as his initial platelet count was dangerously low and the parents could afford the expense. The other three patients who were managed in the state sector did not receive any IVIG partly due to the cost factor and partly as there was no urgency to raise the count before a bone marrow examination was carried out.

All four patients showed a sharp rise in platelet counts within a few days of starting treatment with dexamethasone and on day 5 of the first cycle, all had platelet counts of more than $100 \times 10^{9} / \mathrm{L}$. However, the counts dropped to around 60-80 x $10^{9} / \mathrm{L}$ towards the end of first month in each of the four patients but progressively rose in the successive month to relatively higher levels. All had platelet counts of more than $150 \times 10^{9} / \mathrm{L}$ at the end of the full course of treatment which lasted over a period of six months.

We did not observe any serious side-effects to dexamethasone in any of the four patients. Three showed an increase in appetite while one had temporary hepatomegaly. These changes were more marked in the 1 st month. Their height velocities were not significantly affected.

\section{Discussion}

Patients No.1, 2 and 3 responded well to treatment and have remained in remission up to now. Patient No. 4, who is now a thirteen year old boy, remained in complete remission for almost three years. Unfortunately his platelet count dropped to $43 \times 10^{9} / \mathrm{L}$ in August 2000 and he developed multiple bruises again.

A previous study, done elsewhere, failed to show impressive results with pulsed high dose dexamethasone therapy in children when compared to their adult counterparts ${ }^{4,5}$. However, the duration of treatment in each cycle in the trial under reference had been four days whereas our cycles were of five days duration. This may have contributed to the successful outcome of our trial. Most of the adults have received a relatively higher dose of $20 \mathrm{mg} / \mathrm{m}^{2}$ but we have shown that a relatively lower dose of $15 \mathrm{mg} / \mathrm{m}^{2}$ can be just as effective in bringing about long lasting remission in the case of children provided each cycle lasts for a period of five days.

Three out of the four patients in our series have remained in remission up to now. But we need a further follow up of these patients to see if they will remain in remission forever. As has already been shown in a previous study ${ }^{1}$, we also feel that the duration of disease prior to starting treatment with dexamethasone seems to be related to the outcome. The ten year old boy who relapsed after 
being in remission for almost three years had the illness going on for two years before dexamethasone therapy was begun when compared to the other three patients (mean $0.97 \mathrm{yrs}$ ).

Other modes of treatment such as IVIG are not always practicable owing to financial constraints and even with IVIG relapses are not rare. Immunosuppressive therapy, on the other hand, is not very desirable in children if a safer alternative could be offered. Splenectomy is also not without risk and is known to cause life threatening sepsis, specially in younger children under the age of five years.

\section{Conclusion}

We feel that it is worth giving pulsed high dose dexamethasone therapy for patients with chronic ITP who need some form of intervention before splenectomy is considered. However a multicentre trial involving a wider patient base is desirable before any firm conclusions are drawn with regard to the efficacy of high dose dexamethasone in the treatment of chronic ITP in children.

\section{Acknowledgements}

We sincerely thank the house officers and the nursing staff of Ward 15A Colombo South Teaching Hospital for their assistance.

\section{References}

1. Borgna-Pignalti C, Rugolotto S, Nobili B, Amendola $G$ et al. A trial of high dose dexamethasone therapy for chronic idiopathic thrombocytopenic purpura in childhood. Journal of Pediatrics 1997; 130:13-6.

2. Choudhry V P, Kashyap R, Pati H P. Management of Idiopathic Thrombocytopenic purpura. Indian Journal of Pediatrics 1998; 65: 401-7.

3. Kuhne T, Freedmen J, Seple J W, Doyle J et al. Platelet and Immune responses to oral cyclic dexamethasone therapy in childhood chronic immune thrombocytopenic purpura. Journal of Pediatrics 1997; 130: 17-24.

4. Chen J S, Wu J M, Chen Y J, Yeh T F. Pulsed high dose dexamethasone therapy in children with chronic idiopathic thrombocytopenic purpura. Journal of Paediatric-Haematology Oncology 1997; 19(6): 526-9.

5. Warner M, Wasi P, Couban S, Hayward C et al. Failure of pulsed high dose dexamethasone in chronic idiopathic immune thrombocytopenic purpura. American Journal of Haematology 1997; 54 (4): 267-70. 